\title{
The development of food image detection and recognition model of Korean food for mobile dietary management
}

\author{
Seon-Joo Park ${ }^{1 *}$, Akmaljon Palvanov ${ }^{2 *}$, Chang-Ho Lee ${ }^{3}$, Nanoom Jeong ${ }^{1}$, Young-Im Cho ${ }^{2 \S}$ and Hae-Jeung Lee \\ 'Department of Food and Nutrition, College of BioNano Technology, Gachon University, 1342 Seongnam-daero, Sujeong-gu, Seongnam-si, Gyeonggi 13120, Korea \\ ${ }^{2}$ Department of Computer Engineering, College of IT, Gachon University, 1342 Seongnam-daero, Sujeong-gu, Seongnam-si, Gyeonggi 13120, Korea \\ ${ }^{3}$ Research Group of Functional Food Materials, Korea Food Research Institute, Wanju 55365, Korea
}

BACKGROUND/OBJECTIVES: The aim of this study was to develop Korean food image detection and recognition model for use in mobile devices for accurate estimation of dietary intake.

MATERIALS/METHODS: We collected food images by taking pictures or by searching web images and built an image dataset for use in training a complex recognition model for Korean food. Augmentation techniques were performed in order to increase the dataset size. The dataset for training contained more than 92,000 images categorized into 23 groups of Korean food. All images were down-sampled to a fixed resolution of $150 \times 150$ and then randomly divided into training and testing groups at a ratio of 3:1, resulting in 69,000 training images and 23,000 test images. We used a Deep Convolutional Neural Network (DCNN) for the complex recognition model and compared the results with those of other networks: AlexNet, GoogLeNet, Very Deep Convolutional Neural Network, VGG and ResNet, for large-scale image recognition.

RESULTS: Our complex food recognition model, K-foodNet, had higher test accuracy (91.3\%) and faster recognition time $(0.4$ $\mathrm{ms}$ ) than those of the other networks.

CONCLUSION: The results showed that K-foodNet achieved better performance in detecting and recognizing Korean food compared to other state-of-the-art models.

Nutrition Research and Practice 2019;13(6):521-528; https://doi.org/10.4162/nrp.2019.13.6.521; pISSN 1976-1457 elSSN 2005-6168

Keywords: Food recognition, deep convolutional neural networks (DCNN), mobile device, dietary assessment

\section{INTRODUCTION}

Nutrition surveys have been widely used to assess food and nutrient intake or dietary patterns of a specific target population [1]. Accurate methods to assess food or nutrient intake are necessary to manage daily personal dietary intake and to conduct academic nutritional research. Commonly used dietary assessment methods are $24 \mathrm{~h}$ recall, food frequency questionnaire (FFQ), and food records. Dietary assessments were traditionally completed using Pen-and-Paper interviewing (PAPI) and Computer-assisted Personal Interviewing (CAPI), which are heavily dependent on memory and cognition of the subject [1]. In addition, people may underreport their intake due to the recoding burden [2]. Table 1 is a summary of the features of representative nutrition survey methods.

Recently, a self-report method using a mobile device (e.g., smartphone) has been extensively used to assess dietary intakes. A mobile device camera is used to record meals before and after eating to provide a visual food record [3]. However, major concerns of this approach are associated with food recognition and intake volume (weight) estimation $[4,5]$. However, there are assessment tools available to help people identify their dietary intake. The Wellnavi is a dietary evaluation method based on a portable personal digital assistant device with a phone card and a camera [6,7]. The technology Assisted Dietary Assessment (TADA) image analysis tool is useful for identification and quantification of food intake in which images taken before and after food consumption are used to calculate the amount and type of food consumed [8]. The Remote Food Photography Method (RFPM) uses a smartphone to capture food images before and after consumption, and sends images to a server to provide an estimate of food intake [9]. A multisensor device, the eButton, worn on the chest uses a camera to manually capture all relevant images in front of the subjects [10]. Many of these tools are being improved and are in use in the food intake research field [11]. However, the tools have difficulty recognizing food and estimating the amount of food accurately [12].

\footnotetext{
This work was supported by Korea Food Research Institute (E0164500-04) and Gachon University research fund of 2018 (GCU-2018-0704).

${ }^{\S}$ Corresponding Authors: Hae-Jeung Lee, Tel. 82-31-750-5968, Fax. 82-31-750-5974, Email. skysea@gachon.ac.kr, skysea1010@gmail.com Young-Im Cho, Tel. 82-31-750-5800, Email. yicho@gachon.ac.kr

Received: May 28, 2019, Revised: June 18, 2019, Accepted: August 18, 2019

* These two authors contributed equally to this study.

This is an Open Access article distributed under the terms of the Creative Commons Attribution Non-Commercial License (http://creativecommons.org/licenses/by-nc/3.0/) which permits unrestricted non-commercial use, distribution, and reproduction in any medium, provided the original work is properly cited.
} 
Table 1. Features of representative nutrition survey methods

\begin{tabular}{lll}
\hline \multicolumn{1}{c}{ Method } & Pros \\
\hline $\begin{array}{l}\text { PAPI (Pen and paper interviewing) } \\
\text { CAPI (Computer-assisted personal } \\
\text { interviewing) }\end{array}$ & Low cost \& short time & Consumed lots of time and manpower to recoding research data \\
Mobile device + Dietitian & Less burden of recall & $\begin{array}{l}\text { Computer, PDA, etc. need tools } \\
\text { Program development cost is high }\end{array}$ \\
Mobile device using photographs & $\begin{array}{l}\text { Real-time analysis of nutrients in food intake and Metadata required for accurate nutritional assessment } \\
\text { feedback available }\end{array}$ & $\begin{array}{l}\text { Food photo } \rightarrow \text { Dietitian analyzes and feedback } \\
\text { Image recognition algorithm and system need }\end{array}$ \\
\hline
\end{tabular}

Currently, in Korea, PAPI or CAPI-based nutrition surveys have been performed, but there is a growing demand for mobile device-based dietary assessment tools. However, Asian foods including Korean foods, include a greater variety of cooking methods and a higher number of ingredients than those in Western foods [13]. Furthermore, some similar Korean foods may look different while some entirely different Korean foods look very similar, which makes the identification of food items from images difficult [14].

Most dietary assessment methods using mobile devices have been used by dietitians to estimate intakes using food photographs taken before and after the meal [15-17]. Many approaches to improving dietary intake amount estimation have been proposed [18-20]. The vast majority of those techniques rely on hand-engineered features and traditional signal processing methods. For instance, DietCam [21] uses fusion of a nearest-neighbor based best match search and the SIFT-based Bag of Visual Words (BoW) classification methods to estimate daily food intake. Another food recognition system aimed to estimate the calorie and nutrition levels of foods [22]. The authors used segmented food item regions in order to increase the accuracy of their recognition system: a system that recognized foods based on information sources including the SURF-based Bag of Features and a color histogram extracted from the segmented food item regions. In addition, artificial Intelligence (Al)-based algorithms have been used to detect food items from images obtained from a wearable device [23]. For instance, the Deep Convolutional Neural Network (DCNN), a state-of-the-art technology, has been reported to have reliable results even on large and diverse image datasets with non-uniform image backgrounds [24]. The DCNN to recognize images and it provided accurate detection of food items [24].

The purpose of this study was to evaluate the applicability of food recognition model using DCNN in Korean food items. We undertook automatic Korean food classification using a new DCNN model that recognizes given images of Korean dishes and compared our results using various classification tasks with those from other popular models.

\section{MATERIALS AND METHODS}

\section{Building a dataset for Korean food recognition}

A localized image dataset is required to obtain accurate food item recognition results since foods vary by region [14]. To the best of our knowledge, there is no publicly available image dataset for Korean food item recognition. Therefore, we collected more than 4,000 food images by taking pictures of dishes in restaurants as well as by searching the Internet for web-based images. Sample images from the collected dataset are shown in Fig. 1. In order to build a dataset suitable for training the complex recognition model for Korean food images, we established 23 food groups based on the frequently consumed food list of the Korean National Health and Nutrition Examination Survey; the selected food class names are provided in Table 2.

Since all images were collected from various sources, their format, resolution, and quality were different. Moreover, even though the number of collected images was more than 4,000,

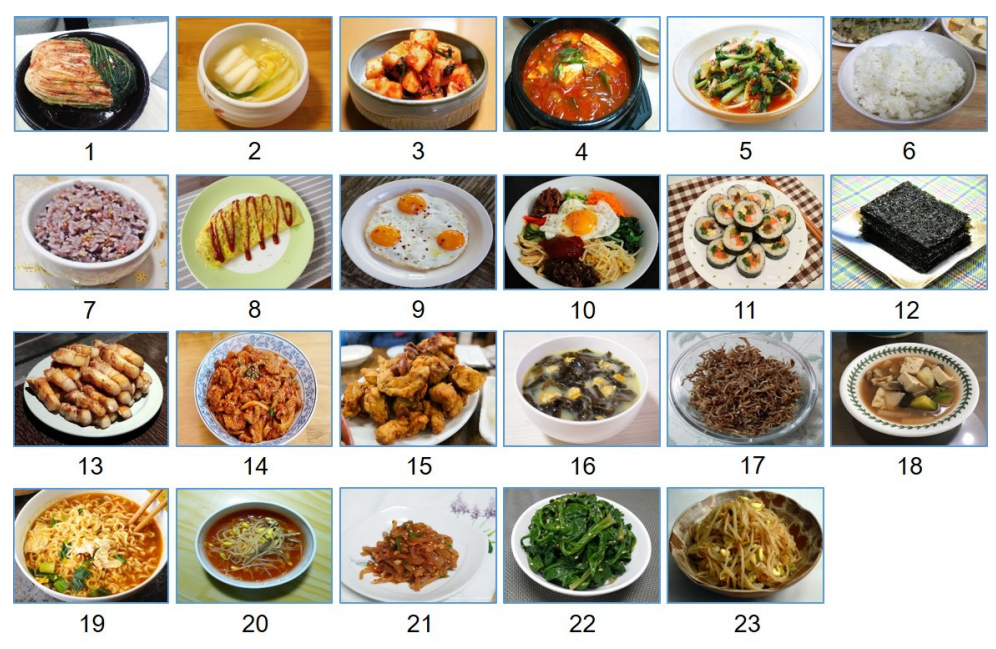

Fig. 1. Sample images of the 23 Korean food groups images unsed in the food recognition dataset 
Table 2. Names of the twenty-three food groups included in the dataset

\begin{tabular}{|c|c|c|c|}
\hline Number & Class name & Number & Class name \\
\hline 1 & Kimchi & 13 & Grilled pork belly \\
\hline 2 & White kimchi & 14 & Stir-fried pork \\
\hline 3 & Radish kimchi & 15 & Fried chicken with sweet and sour source \\
\hline 4 & Kimchi stew & 16 & Seaweed soup \\
\hline 5 & Yeolmu-kimchi & 17 & Stir-fried anchovies \\
\hline 6 & Boiled Rice & 18 & Soybean paste soup \\
\hline 7 & Boiled Rice with multi-grains & 19 & Ramen \\
\hline 8 & Omelet with rice & 20 & Bean sprouts soup \\
\hline 9 & Fried egg & 21 & Dried radish slices seasoned with soy sauce and spices \\
\hline 10 & Bibimpab & 22 & Seasoned spinach \\
\hline 11 & Gimbap & 23 & Seasoned bean sprouts \\
\hline 12 & Grilled seaweed & & \\
\hline
\end{tabular}

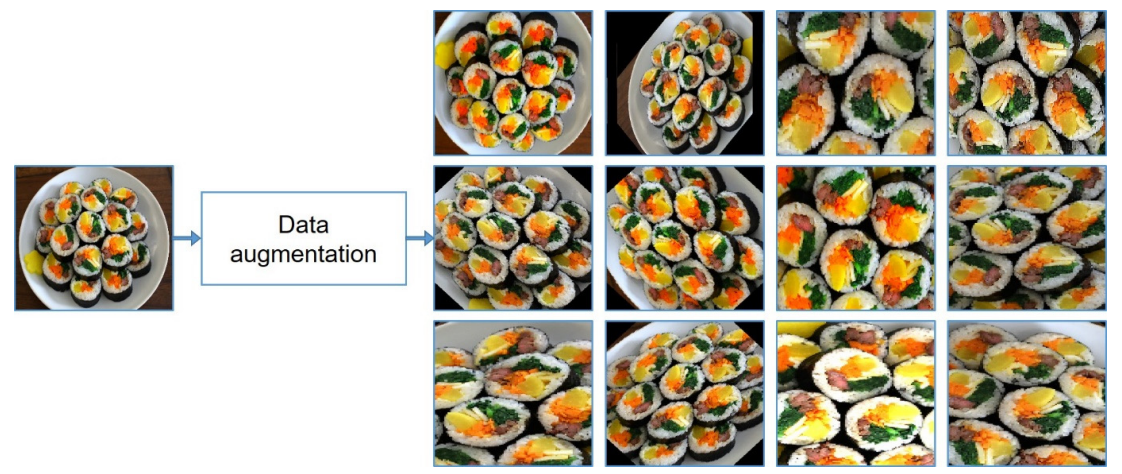

Fig. 2. Examples of artificially generated images used in the for food recognition dataset. Left: a single input image used as the basis for the generation of new images. Right: Artificially generated images obatined by using data augmentation and image processing techniques.

it was insufficient for use in training high quality deep-learning models. Therefore, to increase the dataset size, data augmentation and image processing techniques were performed on more than 4,000 collected images. Data augmentation methods were used to generate new images from a single image whereas the image processing techniques were used for improving the quality and reducing the similarity of the newly generated images. During data generation, random contrast, brightness, sharpness and color changes were added to each of the augmented images in order to decrease image similarity. As a result, the data size substantially increased. Examples of the artificially generated images are presented in Fig. 2. The final dataset contained 92,000 images. After the image collection and image processing were finished, the dataset was prepared for use in the learning process. All images were down-sampled to a fixed resolution of $150 \times 150$ and then randomly divided into training and testing groups at a ratio of $3: 1$, resulting in 69,000 training and 23,000 test images.

\section{K-food network Structure}

A Convolutional Neural Network (CNN) usually consists of convolutional layers and pooling layers [13]. Notations $w$ and $h$ represent width and height, $c h$ is the RGB color channels of the input image $I(w, h, c h)$. the convolutional layer and max-pooling are denoted as $C$ and $M P$, the convolutional layer is $C(k, c s, o)$ and takes kernel size $(k)$, convolutional strides (cs), and the number of output feature maps is (o) as arguments. The pooling layer MP ( $r$, ps) considers the side length of the pooling receptive field $(r)$ and the pooling strides (ps). In addition, $F C$ (c) and $F$ (class) correspond to the fully-connected layer and the output layers, respectively, where (n) is the number of nodes and (c) is one of the food categories. Notation $D$ stands for dropout. All convolutional layers use ReLU as an activation function. The DCNN model $(M)$ is thus represented by:

$$
\begin{aligned}
M \Rightarrow & I(150,150,3) \rightarrow C(9,2,32) \rightarrow C(7,2,64) \rightarrow[C(1,1, \\
& 128), C(3,2,128), C(5,2,128)] \rightarrow \text { concat } \rightarrow[C(1,1, \\
& 128), C(3,2,128), C(5,2,128)] \rightarrow \text { concat } \rightarrow M P(2,2) \\
& \rightarrow D \rightarrow C(3,2,256) \rightarrow M P(2,2) \rightarrow C(3,2,512) \rightarrow M P \\
& (2,2) \rightarrow F C(2048) \rightarrow D \rightarrow F C(2048) \rightarrow F \text { (class). }
\end{aligned}
$$

The Softmax function (normalized exponential function) is defined as:

$$
S(F)_{j}=\frac{\exp ^{F_{j}}}{\sum_{i=1}^{\text {class }} \exp ^{F_{i}}}, \text { for } j=1,2 \ldots, \text { class }
$$

Where, $F_{j}$ is the features of the output layer. The final prediction (Prediction) is gained from the maximum value of $S(F)$

$$
\text { Prediction }=\underbrace{\operatorname{argmax}}_{j}\left(S(F)_{j}\right), \quad \text { for } j=1,2 \ldots, \text { class }
$$




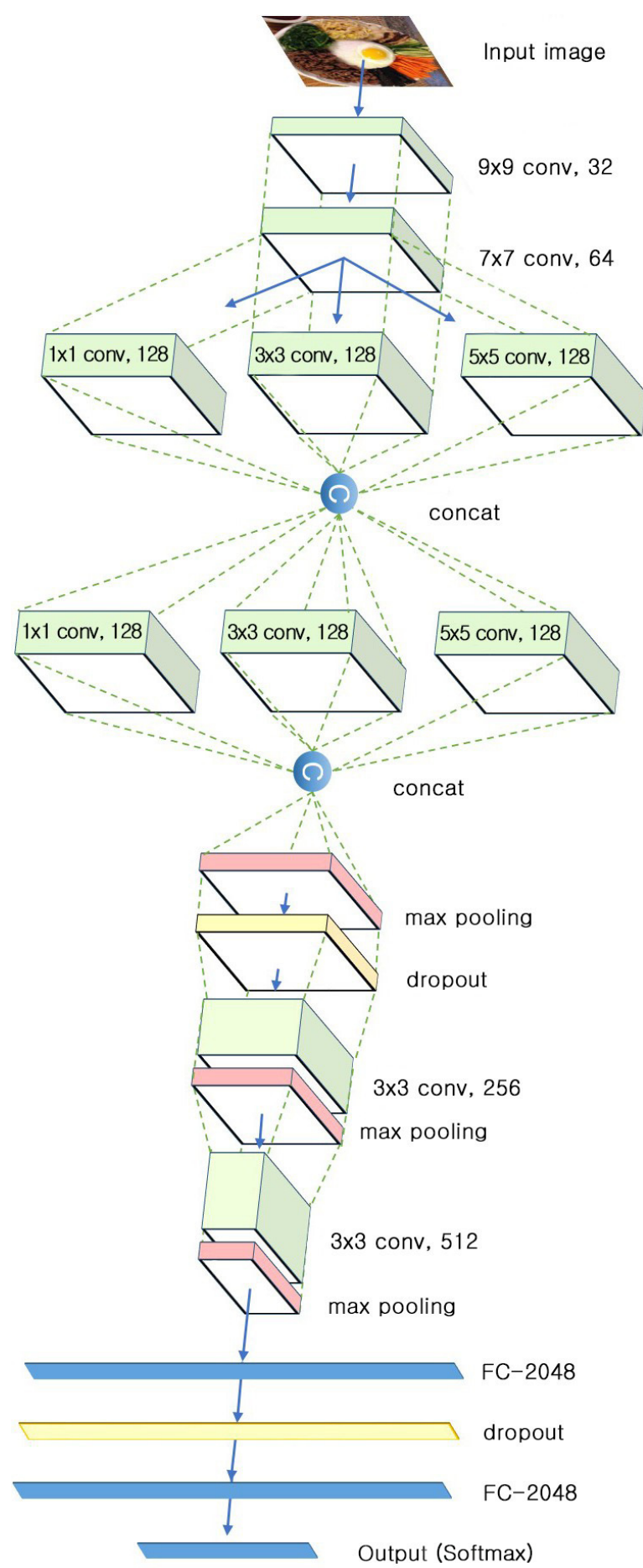

Fig. 3. Schematic architecture of the K-foodNet model that incorporated deep convolutional neural networks.

The schematic architecture of the DCNN used in this study is depicted in Fig. 3. In order to achieve improved performance we implemented a wider and deeper network. The initial layer receives $150 \times 150 \times 3$ tensors (RGB values for a $150 \times 150 \times 3$ image) as an input and produces 32 feature maps by convolving with the kernel size $9 \times 9$. A $7 \times 7$ kernel is used to convolve the previous output, resulting in 64 feature maps.

Subsequently, every map will be simultaneously convolved with $1 \times 1,3 \times 3$ and $5 \times 5$ convolutional kernels, in which, there are three separate layers. Each of these layers will produce 128 feature maps. The output tensors will be concatenated before feeding the next convolution layers with the same kernels. The produced output tensors are down-sampled using a max pooling operation after the second concatenation. The first dropout layer is used after max pooling to reduce overfitting.

Convolutional-pooling layers using $3 \times 3$ kernel and producing 256 and 512 feature maps are followed by the max polling layers. After the last pooling layer, the fully connected (FC) layers are activated with 2048 nodes as well as the dropout layer between the FC layers. All convolutional and FC layers use ReLU activation functions. Finally, there are 23 SoftMax neurons in the output layer, which corresponds to the 23 groups of food.

\section{Experimental settings}

A high-end server with 64GB of RAM and equipped with two Nvidia GeForce GTX 1080 Ti GPUs was used to do the training. The training was carried out by using TensorFlow machine learning framework and a batch size of 64 was established; the batches were randomly shuffled during the training process. In addition, two training schemas (20 and 40 epochs) were implemented to determine the different behaviors of the model. Dropout is used to prevent overfitting in neural networks [13]. The dropout layer was used twice within the model to reduce overfitting and both dropout layers had a 0.4 equal rate during training. We used a placeholder to dynamically control the dropout rate when simultaneous training and inference. The dropout rate was set to turn off the function to make predictions and inferences.

During the training, TensorFlow's AdamOptimizer() [25] function was used as the optimizer while the sparse_softmax_cross entropy() [26] function was used as a loss function. In order to train the DCNN, choosing suitable hyperparameters is essential among those, the leaning rate $(n)$ is the most critical as it significantly affects the training performance. However, the use of a fixed learning rate for the entire training process is not an optimal solution as it does consider the dynamical nature of the training behavior of the model. Therefore, the learning rate was dynamically updated throughout the training process. The function which updated that rate was the exponential function of cost $n=n 0 \times \exp$ (loss), where loss was the value of the sparse_softmax_cross_entropy() obtained during training and $n 0$ was equal to $1 \mathrm{e}^{-4}$. Such a schedule for updating the learning rate strongly is related to the training performance.

The initial speed of the training was high due to the high learning rate, but training loss was also large; subsequently, the learning rate decreased automatically to avoid overshooting the best result.

\section{RESULTS}

\section{Accuracies of $K$-foodNet model}

The graphs in Fig. 4 reveal both training and testing accuracies as well as the loss functions of the model with 20 epochs. The loss function decreased rapidly and maintained a 
(A)

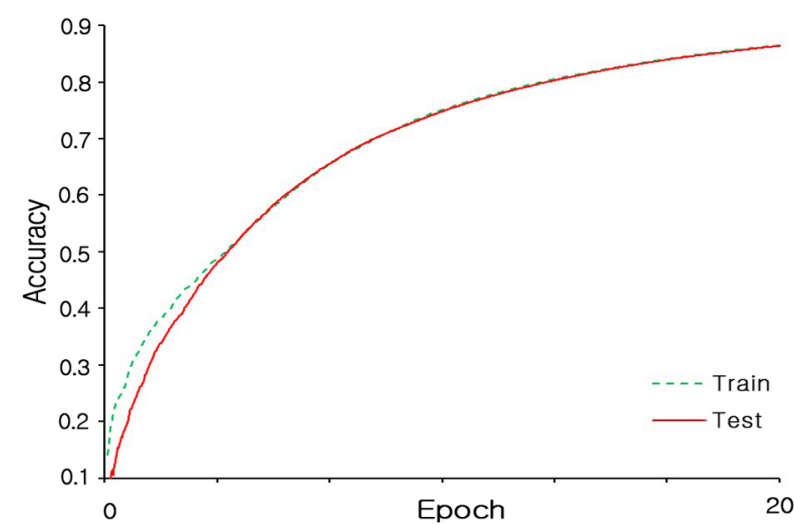

(B)

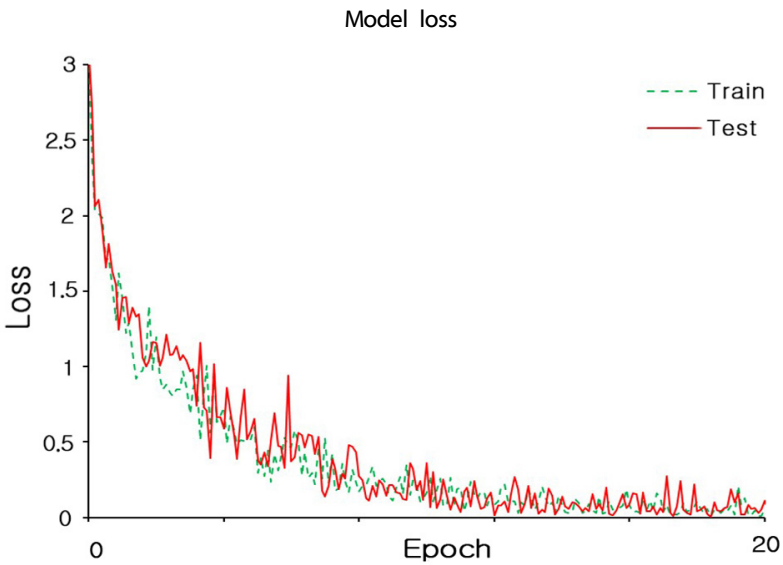

Fig. 4. Learning results of the proposed K-foodNet model when using 20 epochs. (A) Accuracy functions of the model during training and testing. (B) Loss functions of the model during training and testing.

(A)

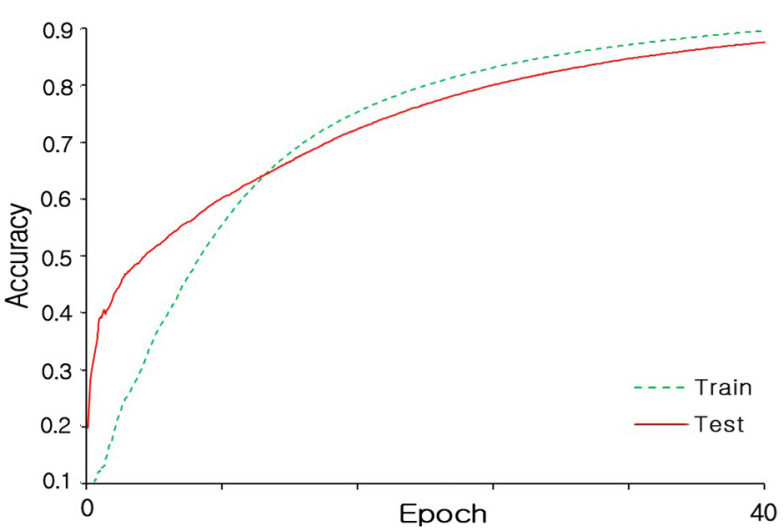

(B)

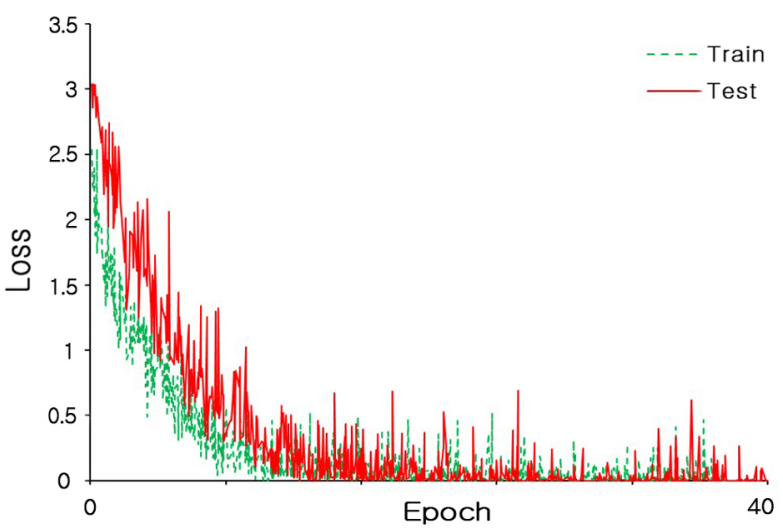

Fig. 5. Learning results of the proposed K-foodNet model when using 40 epochs. (A) Accuracy functions of the model during training and testing. (B) Loss functions of the model during training and testing.

steady index around zero. The test accuracy plateaued at approximately $88 \%$ within just 20 epochs.

Based on the accuracy and loss curves in the Fig. 5, it is clear that the performance of the model could be further improved by increasing the number of training epochs. The figure shows that the gap between test and training functions is not large and that both curves increase over subsequent epochs, during which loss rapidly decreases before maintaining a stable rate in both training and test cases. The use of 40 epochs resulted in a $90 \%$ accuracy in the test, which shows the model could be trained to achieve higher accuracy.

In order to evaluate the performance of the proposed model, we trained the model 20 epochs. Fig. 4 shows that after the training was complete, training and testing accuracy had not stabilized. In other words, there was no overfitting, indicating that the model is still learning. In further experiments, we trained the same network and dataset using the same configurations; however, for that additional training case, we have assigned 40 epochs to determine whether the model still could be improved. Interestingly, as shown Fig. 5, after 40 epochs the model is still improving without overfitting; moreover, the model performance is sufficiently promising for application to an artificially extended dataset.

Comparisons of test accuracies and prediction times of the $K$-foodNet model and existing state-of-the-art models

In order to provide a fair benchmark to our K-foodNet, we trained other models with the same configurations and the same number of epochs (40 epochs) to compare with the results

Table 3. Comparisons of test accuracies and prediction times of the K-foodNet model and existing state-of-the-art models

\begin{tabular}{lcc}
\hline \multicolumn{1}{c}{ Model name } & Test accuracy & Prediction time \\
\hline AlexNet & $71.6 \%$ & $0.46 \mathrm{~ms}$ \\
GoogLeNet & $80.5 \%$ & $0.8 \mathrm{~ms}$ \\
VGG-19 & $79.22 \%$ & $0.57 \mathrm{~ms}$ \\
ResNet-18 & $81.6 \%$ & $1.2 \mathrm{~ms}$ \\
K-foodNet & $91.3 \%$ & $0.42 \mathrm{~ms}$ \\
\hline
\end{tabular}

Number of food categories $=23$

Number of epochs $=40$

Size of input image $=150 \times 150$

Number of training images $=69,000$

Number of testing images $=23,000$ 


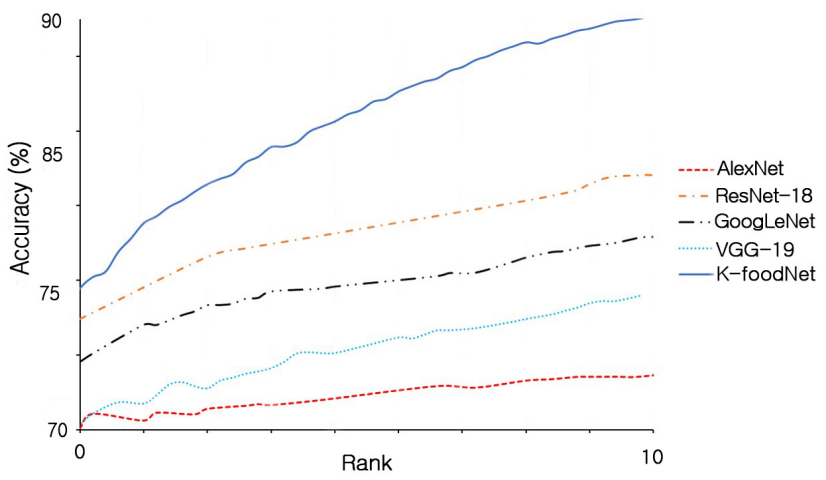

Fig. 6. Performance accuracy of the K-foodNet model compared to a variety of existing state-of-the-art models.

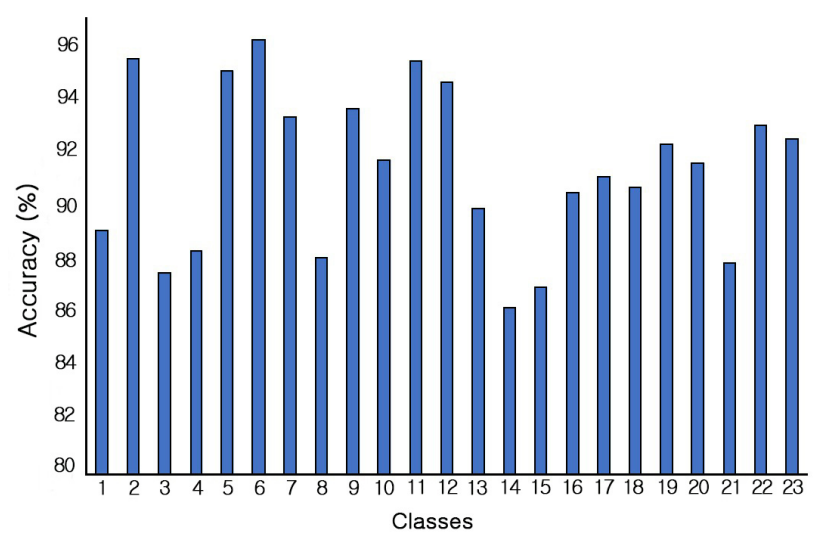

Fig. 7. Average test accuracy for each of the 23 Korean food groups. 1 Kimchi 2 White kimchi 3 Radish kimchi 4 Kimchi stew 5 Yeolmu-kimchi 6 Boiled Rice 7 Boiled rice with multi grains 8 Omelet with rice 9 Fried egg 10 Bibimbap 11 Gimbap 12 Grilled seaweed 13 Grilled pork belly 14 Stir-fried pork 15 Fried chicken with sweet and sour source 16 Seaweed soup 17 Stir-fried anchovies 18 Soybean paste soup 19 Ramen 20 Bean sprouts soup 21 Dried radish slices seasoned with soy sauce and spices 22 Seasoned spinach 23 Seasoned bean sprouts.

of our method. A summary of the comparisons is provided in the Table 3. Among the tested networks, our model was the fastest in term of prediction. In addition, the other models were deeper than our network but suffered from overfitting. Finally, the overall test accuracy of our method was higher than that of the other models.

The Fig. 6 illustrates the performance accuracy between K-foodNet and existing state-of-the-art models. It is obvious in Fig. 6 that our method consistently outperformed all of the current state-of-the-art networks when examining our new datasets.

\section{Average test accuracy of the 23 Korean food groups}

Fig. 7 illustrates the results of the average testing accuracy for each of the 23 individual food groups. Among those groups, the model's classification accuracies for images of white Kimchi, boiled rice, and Gimbap were more than 95\%. However, the classification accuracies for stir-fried pork and radish Kimchi were approximately $87 \%$, indication that these latter two food groups were more difficult to recognize.

\section{DISCUSSION}

Food intake assessment has important roles in chronic diseases management and the provision of public healthcare services. Recently, there has been a growing demand for nutrition management via mobile device applications. Mobile phones have been considered the most effective tool for gathering and delivering food information [21]. Thus far, many mobile device tools and programs have been developed and used to estimate food intake. For example, DietCam, an automatic food calorie measurement system, was developed for use in obesity nutrition management [12]. DietCam consists of three parts: image management, food volume estimation, and food classification. It uses optical character recognition (OCR) techniques and a scale-invariant feature transform (SIFT). A feature-based food classification approach and a multi-view method to calculate the food calorie and the volumes has also been described [21]. In addition, a wearables device, an automatic ingestion monitor, and a neural network classifier have been used to detect and monitor food intake of participants at a resolution of $30 \mathrm{~s}$ [27]. NutriNet, which is based on the recognition of food images by using DCNN, was developed as a dietary assessment applicaion for Parkinson's patients [14]. DeepFood is a food image recognition system that uses deeplearning algorithms to evaluate dietary intake [28]. These provious studies and the associated investigative tools were undertaken to accurately recognize food images and volumes by applying various algorithms and deep-learning methods, but prior to this study, no such studies have been undertaken in Korea.

A novel DCNN model based on real-time recognition of digital Korean food images was implemented in this study. The new dataset consisted of popular Korean food images and contained more than 4,000 original images in 23 food groups and the images illustrated mostly common dishes consumed in daily life in Korea. After expanding the dataset via data augmentation, the acquired number of images was more than 92,000. We applied DCNN in the complex recognition model and compared the result with other large-scale image recognition networks: AlexNet, GoogLeNet, Very Deep Convolutional Neural Network, VGG and ResNet, for large-scale image recognition. Our study results showed K-foodNet achieved better performance in detecting and recognizing Korean food compared to other state-of-the-art models.

To our best knowledge, there has never been a DCNN-based food recognition algorithm developed for Korean food. One of the challenges we faced was the unique characteristics of Korean foods [13]. Input images were different in terms of shape, texture, size and color as the Korean foods lack a typical or generalized layout. Korean foods are more complex than other types of food such as Indian or Italian food, and recognition of images of Korean food is difficult because images of foods within the same food category can appear different. In general, Korean food may be cooked with different ingredients and using different cooking methods; thus, images of the same food item can look dissimilar. Moreover, the same food item can look different to the naked eye. In addition, image noise from various backgrounds and textures is an obstacle to 
recognition as all images in the dataset were captured in a variety of places and environments, thereby including insufficient or different image brightness, strong reflection, distracting ornaments, etc.

Our approach had several strengths when compared to the aforementioned image recognition approaches. First, we have designed a new model on the assumption that CNNs that can handle a large artificially extended dataset of food images. More images in our dataset were generated from a limited number of images by using data augmentation techniques; therefore, similarity was very high between images, which, in turn, can lead to strong overfitting during training. However, it is clear from the results that the model is stable and is capable of learning images without overfitting. Although the above mentioned approaches use classic off-the-shelf deep learning architectures, our novel solution produced better performance and robustness with respect to existing approaches. Furthermore, our model is able to accurately distinguish images with very complex textures, in other words, images that belong to the same food category can appear very different due to differences in texture. In spite of these challenges, our model achieved excellent results compared to other currently available models. Others have noted that in computer vision tasks, CNN-based models can outperform traditional methods and achieve higher accuracy when using deeper CNNs $[14,29,30]$. A model purposed by Lu [31], obtained an overall $90 \%$ accuracy using DCNNs and a small dataset with 5,822 images and 10 food groups. Their model used five convolutional layers to recognize food images. Moreover, a DCNN-based model, FoodNet, was proposed by Pandey et al. [32]. In that model, the dish image recognition system used a large dataset (ETH Food-101) that include 101 food categories.

Although our model achieves high performance, its loss function is considered too noisy and not capable of a smooth reduction due to the use of similar augmented images. This problem requires future study to resolve. A possible solution to such a problem would be to use reduce the network depth and/or to remove very similar food images from the dataset.

The limitations of this study are as follows. First, the limited number of high quality, specific food images. Second, although a wide range of food categories exists in Korean food, we only included 23 groups of Korean food. Third, there is no publicly available image dataset suitable for Korean food recognition in Korea. Importantly, the quality of images in a dataset has a pivotal role in training the $\mathrm{DCNN}$, and obtaining high performance from deep models is still data-driven to some extent. Therefore, in order to improve the performance of the current model, high-quality images obtained under sufficient lighting conditions and from appropriate angles are needed. As well, the food should be presented in an appropriate, recognizable manner.

The next step in this research is to improve the DCNN algorithm's food image recognition performance level and ensure that the recognition process uses high quality, appropriate images. Such improvements will produce a model that can assess dietary intake accurately and be applied to nutrition management programs in Korea.

\section{ACKNOWLEDGMENTS}

We thank Ms.Yeonjae Lee for her contribution to grammar correction and proofreading.

\section{CONFLICT OF INTEREST}

The authors declare no potential conflicts of interests.

\section{ORCID}

Seon-Joo Park: https://orcid.org/0000-0002-1825-1815

Akmaljon Palvanov: https://orcid.org/0000-0002-4590-2484

Changho Lee: https://orcid.org/0000-0002-1039-1434

Nanoom Jeong: https://orcid.org/0000-0002-4210-7143

Young Im Cho: https://orcid.org/0000-0003-0184-7599

Hae-Jeung Lee: https://orcid.org/0000-0001-8353-3619

\section{REFERENCES}

1. De Keyzer W, Bracke T, McNaughton SA, Parnell W, Moshfegh AJ, Pereira RA, Lee HS, van't Veer $P$, De Henauw $S$, Huybrechts I. Cross-continental comparison of national food consumption survey methods--a narrative review. Nutrients 2015;7:3587-620.

2. Johnson RK, Soultanakis RP, Matthews DE. Literacy and body fatness are associated with underreporting of energy intake in US low-income women using the multiple-pass 24-hour recall: a doubly labeled water study. J Am Diet Assoc 1998;98:1136-40.

3. Stumbo PJ. New technology in dietary assessment: a review of digital methods in improving food record accuracy. Proc Nutr Soc 2013;72:70-6.

4. Martin CK, Kaya S, Gunturk BK. Quantification of food intake using food image analysis. Conf Proc IEEE Eng Med Biol Soc 2009; 2009:6869-72.

5. Lazarte $C E$, Encinas ME, Alegre C, Granfeldt Y. Validation of digital photographs, as a tool in 24-h recall, for the improvement of dietary assessment among rural populations in developing countries. Nutr J 2012;11:61.

6. Kikunaga S, Tin T, Ishibashi G, Wang DH, Kira S. The application of a handheld personal digital assistant with camera and mobile phone card (Wellnavi) to the general population in a dietary survey. J Nutr Sci Vitaminol (Tokyo) 2007;53:109-16.

7. Wang DH, Kogashiwa M, Ohta S, Kira S. Validity and reliability of a dietary assessment method: the application of a digital camera with a mobile phone card attachment. J Nutr Sci Vitaminol (Tokyo) 2002;48:498-504.

8. Khanna N, Boushey CJ, Kerr D, Okos M, Ebert DS, Delp EJ. An overview of the technology assisted dietary assessment project at Purdue University. ISM 2010:290-5.

9. Martin CK, Correa JB, Han H, Allen HR, Rood JC, Champagne CM, Gunturk BK, Bray GA. Validity of the remote food photography method (RFPM) for estimating energy and nutrient intake in near real-time. Obesity (Silver Spring) 2012;20:891-9.

10. Beltran A, Dadabhoy H, Ryan C, Dholakia R, Jia W, Baranowski J, Sun M, Baranowski T. Dietary assessment with a wearable camera among children: feasibility and intercoder reliability. J Acad Nutr Diet 2018;118:2144-53.

11. Wang $\mathrm{DH}$, Kogashiwa $\mathrm{M}$, Kira S. Development of a new instrument 
for evaluating individuals' dietary intakes. J Am Diet Assoc 2006;106:1588-93.

12. He H, Kong F, Tan J. DietCam: multiview food recognition using a multikernel SVM. IEEE J Biomed Health Inform 2016;20:848-55.

13. Zhang XJ, Lu YF, Zhang SH. Multi-task learning for food identification and analysis with deep convolutional neural networks. J Comput Sci Technol 2016;31:489-500.

14. Mezgec S, Koroušić Seljak B. NutriNet: a deep learning food and drink image recognition system for dietary assessment. Nutrients 2017;9:9.

15. Chang UJ, Ko SA. A study on the dietary intake survey method using a cameraphone. Korean J Community Nutr 2007;12:198-205.

16. Son HR, Lee SM, Khil JM. Evaluation of a dietary assessment method using photography for portion size estimation. J Korean Soc Food Cult 2017;32:162-73.

17. Jung $H$, Yoon J, Choi KS, Chung SJ. Feasibility of using digital pictures to examine individuals' nutrient intakes from school lunch: a pilot study. J Korean Diet Assoc 2009;15:278-85.

18. Matsuda $Y$, Yanai K, editors. Multiple-food recognition considering co-occurrence employing manifold ranking. Proceedings of the $21 \mathrm{st}$ International Conference on Pattern Recognition; 2012 Nov 11-15; Tsukuba, Japan. Piscataway: IEEE; 2013 Feb. 2017-20 p.

19. Zhu F, Bosch M, Woo I, Kim S, Boushey CJ, Ebert DS, Delp EJ. The use of mobile devices in aiding dietary assessment and evaluation. IEEE J Sel Top Signal Process 2010;4:756-66.

20. Daugherty BL, Schap TE, Ettienne-Gittens R, Zhu FM, Bosch M, Delp EJ, Ebert DS, Kerr DA, Boushey CJ. Novel technologies for assessing dietary intake: evaluating the usability of a mobile telephone food record among adults and adolescents. J Med Internet Res 2012;14:e58.

21. Kong F, Tan J. DietCam: automatic dietary assessment with mobile camera phones. Pervasive Mob Comput 2012;8:147-63.

22. Kawano $Y$, Yanai $K$, editors. Real-time mobile food recognition system. 2013 IEEE Conference on Computer Vision and Pattern Recognition Workshops; 2013 Jun 23-28; Portland, USA. Piscataway: IEEE; 2013 Sep. 1-7 p.

23. Jia W, Li $Y$, Qu R, Baranowski T, Burke LE, Zhang H, Bai $Y$, Mancino $J M, X u G$, Mao ZH, Sun M. Automatic food detection in egocentric images using artificial intelligence technology. Public Health Nutr 2019;22:1168-79.
24. Kagaya $H$, Aizawa $K$, Ogawa $M$, editors. Food detection and recognition using convolutional neural network. Proceedings of the 22nd ACM International Conference on Multimedia; 2014 Nov 3-7; Orlando, USA. New York: ACM; 2014 Nov. 1085-8 p.

25. Kingma DP, Ba J. Adam: a method for stochastic optimization. Published as a Conference Paper at the 3rd International Conference for Learning Representations; 2015 May 7-9; San Diego, USA. La Jolla: ICLR; 2017 Jan. 1-15 p.

26. Pang T, Xu K, Dong Y, Du C, Chen N, Zhu J. Rethinking softmax cross-entropy loss for adversarial robustness [Internet]. Ithaca (NY): Cornell University; 2019 [cited 2019 June 26]. Available from: https://arxiv.org/abs/1905.10626.

27. Farooq M, Doulah A, Parton J, McCrory MA, Higgins JA, Sazonov E. Validation of sensor-based food intake detection by multicamera video observation in an unconstrained environment. Nutrients 2019;11:E609.

28. Liu C, Cao Y, Luo Y, Chen G, Vokkarane V, Ma Y, editors. DeepFood: deep learning-based food image recognition for computer-aided dietary assessment. ICOST 2016 Proceedings of the 14th International Conference on Inclusive Smart Cities and Digital Health; 2016 May 25-27; Wuhan, China. Cham: Springer International Publishing; 2016 May. 37-48 p.

29. Hassannejad H, Matrella G, Ciampolini P, De Munari I, Mordonini $\mathrm{M}$, Cagnoni $\mathrm{S}$, editors. Food image recognition using very deep convolutional networks. Proceedings of the 2nd International Workshop on Multimedia Assisted Dietary Management; 2016 Oct 16; Amsterdam, The Netherlands. New York: ACM; 2016 Oct. 41-9 p.

30. Christodoulidis S, Anthimopoulos M, Mougiakakou S, editors. Food recognition for dietary assessment using deep convolutional neural networks. New Trends in Image Analysis and Processing -- ICIAP 2015 Workshops; 2015 Sep 7-8; Genova, Italy. Cham: Springer International Publishing; 2015 Aug. 458-65 p.

31. Lu Y. Food image recognition by using convolutional neural networks (CNNs) [Internet]. Ithaca (NY): Cornell University; 2019 [cited 2019 May 28]. Available from: https://arxiv.org/abs/1612. 00983.

32. Pandey P, Deepthi A, Mandal B, Puhan NB. FoodNet: recognizing food using ensemble of deep networks. IEEE Signal Process Lett 2017;24:1758-62. 